\title{
Bulk Silicon Micro-Machined MEM Switches For Millimeter-Wave Applications
}

\author{
K. Grenier ${ }^{1,2}$, P. Pons ${ }^{1}$, R. Plana ${ }^{1,2}$, J. Graffeuil $^{1,2}$ \\ ${ }^{1}$ LAAS / CNRS, 7 av. du Colonel Roche, 31077 TOULOUSE cedex 4, France \\ ${ }^{2}$ Université Paul Sabatier, 118 Route de Narbonne, 31062 Toulouse cedex 4, France
}

\begin{abstract}
This paper deals with the realization of micro-electro-mechanical (MEM) components dedicated to microwave and millimeter-wave applications and focuses on the advantage of using bulk silicon micro-machining. The silicon substrate etching benefits have been demonstrated through both electromagnetic simulations and measurements of a single MEM switch. Important improvements in term of insertion loss and isolation have been obtained. Further developments in term of distributed switches are addressed.
\end{abstract}

\section{INTRODUCTION}

In order to satisfy the future communication systems requirements, new components such as Micro-Electro-Mechanical Systems (MEMS) are nowadays investigated. In the opposite of structures based on the use of active devices, microwave MEMS feature very low insertion loss and power consumption, no problem of linearity, distortion or intermodulation. These kinds of structures would also accommodate high switching power.

Nevertheless, all the previous MEM switches already published [1-4] are limited in term of isolation. In order to improve this performance together with the insertion loss reduction, we have investigated the use of a bulk micro-machining of our silicon substrate to get suspended circuits on membrane.

In this paper, we firstly address (section II) the basics of MEMs switches achieved through a technology based on both surface and bulk micromachinings of silicon. Section III describes the design methodology that has been implemented to synthesize single switches. In section IV, both simulated and measured results obtained on a single micro-machined switch are discussed and compared with respect to those obtained on an other switch processed on a bulk high resistivity silicon (HRS). Finally computed results obtained on a distributed switch are reported. Conclusions are outlined in section $\mathrm{V}$.

\section{MEM SWITCH BASICS}

Our investigated demonstrators consist in capacitive switches, based on the use of electrostatically actuated bridge. Figure 1 shows three of them arranged as a distributed switch.
By applying a DC voltage on the central conductor of the coplanar waveguide (CPW) transmission line, the bridges are attracted towards the line thanks to the electrostatic force. The bridges are pulled down for a voltage called Vp (V pulldown).

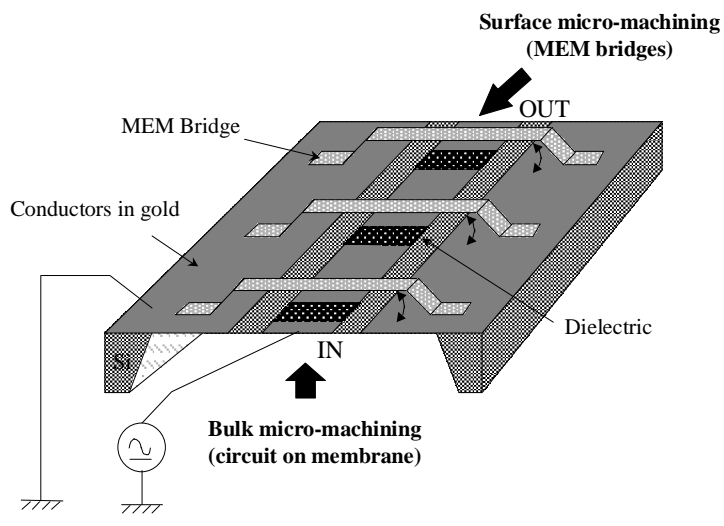

Figure 1. Schematic of a micro-machined MEM distributed switch

When the bridges are up, no DC voltage being applied on the conductor, the microwave signal is fully transmitted. This situation corresponds to the 'ON' state. When the applied DC voltage pulls down the bridges, an ac short takes place between the central conductor of the line and the ground planes. The transmission is thus interrupted, which represents the 'OFF' state.

The corresponding technological process involves a real challenge, as both surface and bulk micro-machinings are required. The surface micromachining is needed to obtain the movable air bridges, whereas the bulk micro-machining of the silicon substrate is used to get circuits with low insertion losses. To avoid any DC short in the OFF state, a dielectric layer deposited on the CPW signal line beneath the bridge is required. Details of the technological process could be found in [5-6].

Next section will focus on the methodology used to design MEM switches. 


\section{SWITCH DESIGN METHODOLOGY}

To design a MEM switch [7], we can consider its simplified electrical equivalent model shown in figure 2 .

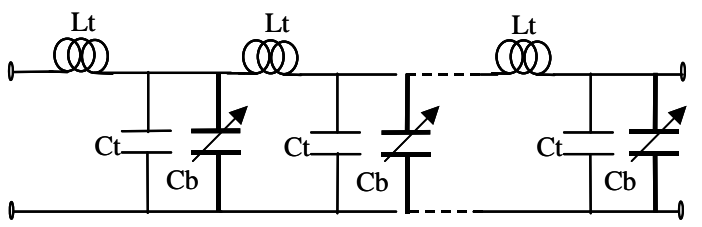

Figure 2. Simplified electrical model of a distributed switch

$\mathrm{Lt}$ and $\mathrm{Ct}$, respectively the inductor and capacitor per unit length, refers to the CPW line without any bridge, whereas the variable capacitor $\mathrm{Cb}$ refers to the bridge extra capacitance that loads periodically the $\mathrm{CPW}$ line as a first approximation.

The characteristic impedance of a distributed switch is therefore given by the equation (1) :

$$
\mathrm{Zc}=\sqrt{\frac{\mathrm{Lt}}{\mathrm{Ct}+\mathrm{Cb} / \mathrm{s}}}
$$

where $\mathrm{s}$ is the periodical space between the bridges.

When the bridges are down, the characteristic impedance dramatically decreases since the value of the bridge capacitor $\mathrm{Cb}$ is very large. Most of the signal is therefore reflected and a very low transmission occurs.

When the bridges are up, $\mathrm{Cb}$ is small and it translates into a finite value of $\mathrm{Zc}$ that depends also on the CPW dimensions. To achieve the best transmission in this state, $\mathrm{Zc}$ has to be equal to the reference impedance (chosen here as to be $75 \Omega$ ). A detailed investigation of the MEM bridge electrical behavior is consequently needed to determine its capacitor.

To that end, an equivalent RLC circuit [4] model has been used. Electromagnetic simulations (using SONNET ${ }^{\mathrm{TM}}$ and its air bridge simulation capabilities) have provided us with the electrical elements of the model for different dimensions of bridges and coplanar lines. An electrical simulation and optimization (using $\mathrm{ADS}^{\mathrm{TM}}$ ) was finally completed to get the appropriate dimensions of the bridge and of the CPW.

Next section will be dedicated to some comments on the electromagnetic simulations and on the measurements performed on single bridges.

\section{RESULTS}

\section{A. Single MEM bridge}

From electromagnetic simulations of a single MEM bridge, we can observe the current density along the CPW beneath the bridge in the OFF state as shown in figures 4 and 5 for both types of substrate on suspended membrane and on HRS respectively.

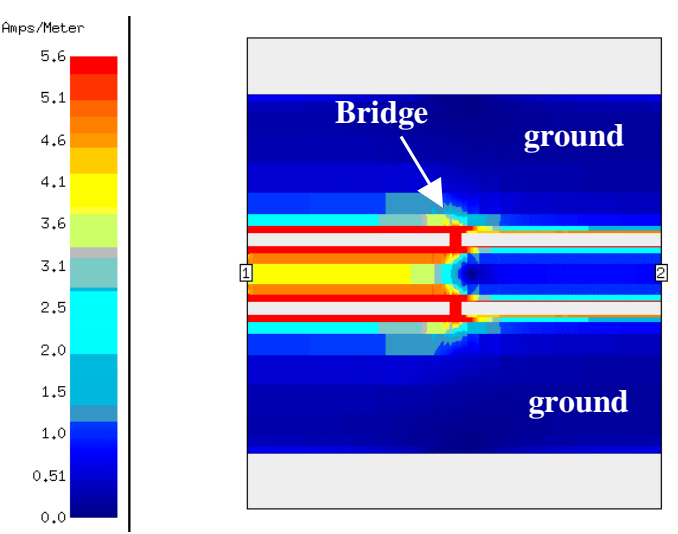

Figure 4. Current density view of a MEM bridge on a suspended membrane

As expected, almost all the signal is shorted when the bridge is down (the current is dramatically reduced at the right end of the picture) and most of the current density is localized along the sides of the coplanar slots on the left side.
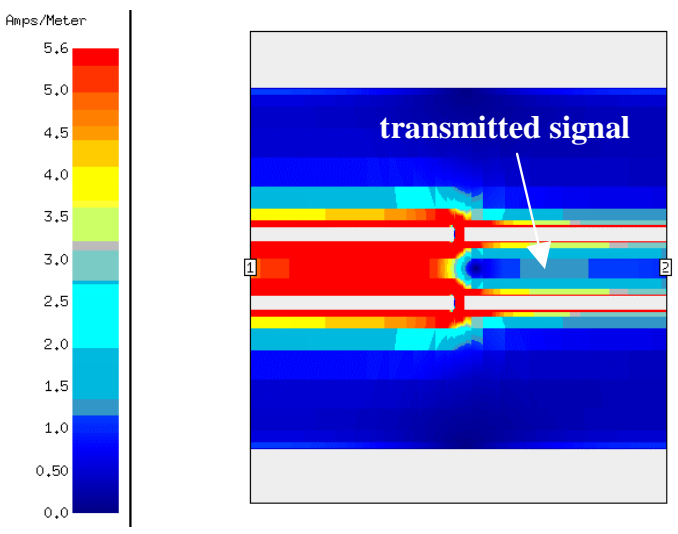

Figure 5. Current density view of a MEM bridge on HRS

But, if we compare figures 4 and 5 , we can notice that more current density is present at the right end of the structure on HRS than on membrane. It is therefore expected that a MEM structure on bulk substrate will feature a better performance in term of isolation than a bulk micromachined one. In fact, the micro-machining of the substrate reduces the input-output coupling through the substrate and thus increases the isolation. To verify this expectation, we have processed two single bridges (with and without bulk micromachining) and measured them in the $\mathrm{ON}$ and OFF states.

The measured data in the OFF state of the bridge are reported in figure 6 . It can be observed that the isolation obtained for the micro-machined structure is $28 \mathrm{~dB}$ at $10 \mathrm{GHz}$ compared to only 23 $\mathrm{dB}$ for the bridge on HRS.

When no voltage is applied on the central conductor of the line ('ON' state), the obtained 
insertion losses are very small, especially in the case of the suspended circuit. At $10 \mathrm{GHz}$, the insertion loss is $0.025 \mathrm{~dB}$ only for the circuit on membrane, whereas the bridge on HRS exhibits a $0.33 \mathrm{~dB}$ insertion losses $(\tan \delta=0.018)$.

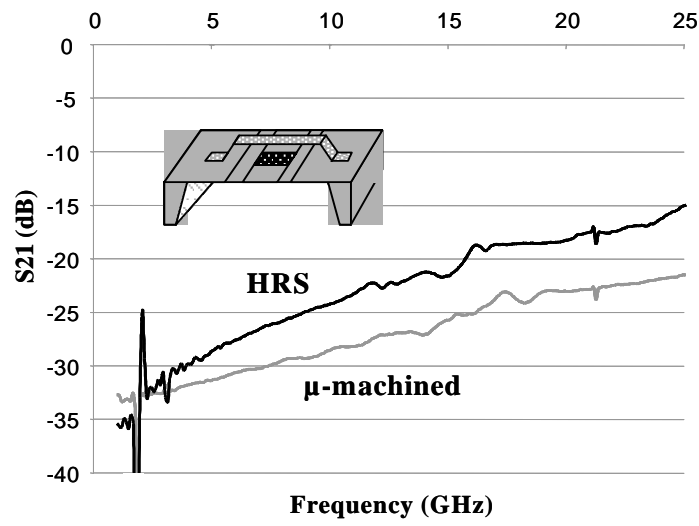

Figure 6. Frequency evolution of the transmission of a single bridge in the down state

These data confirm the previous results issued from electromagnetic simulations (current density distribution) and demonstrate the advantage of suspended structures.

\section{B. Distributed switch with 4 MEM bridges}

In order to further validate the interest of using bulk micro-machining in the realization of MEMS, we have also achieved the whole electromagnetic simulation and optimization of a distributed switch composed of 4 bridges periodically spaced. The correspondent simulated optimized performances are given in figure 7 for the OFF state.

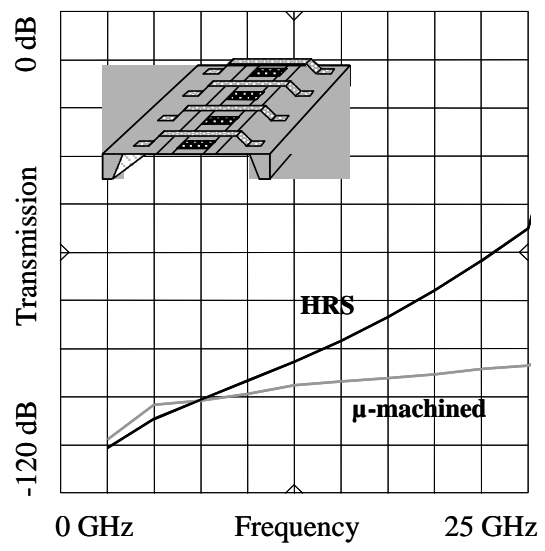

Figure 7. Simulated frequency evolution of a distributed switch in the down state

Thanks to the substrate's micro-machining, a very high isolation level (about $90 \mathrm{~dB} @ 25 \mathrm{GHz}$ ) can be theoretically reached. Although such a value is not totally realistic since input-output coupling through air is not totally accounted for in our simulations, we expect to demonstrate a measured isolation in excess of $50 \mathrm{~dB} @ 25 \mathrm{GHz}$ which is impossible to achieve with usual switches on bulk substrate or with active devices.

We can also observe that the isolation level of the switch on HRS greatly increases versus frequency. Thus the frequency range of suspended high isolation MEM structures can be easily extended in the millimeter-wave range whereas structures on HRS will not provide a large isolation beyond 25 to $30 \mathrm{GHz}$.

\section{CONCLUSIONS}

Benefits of realizing suspended silicon micromachined MEMS switches for microwave and millimeter-wave applications have been demonstrated. Electromagnetic simulations of switches, with only one MEM bridge, and measurements of the previous device have evidenced the better isolation level (28 $\mathrm{dB} @ 10 \mathrm{GHz})$ and lower insertion loss $(0.025 \mathrm{~dB}$ $@ 10 \mathrm{GHz})$ obtained with the micro-machined structure with respect to the similar structure on HRS. Preliminary computed performance of a distributed switch composed of 4 MEM bridges have highlighted the high isolation level that can be expected (more than $50 \mathrm{~dB} @ 25 \mathrm{GHz}$ ), thanks to the micro-machining.

Moreover, the bulk micro-machining of the substrate could not only be used in the realization of switches alone but also extended towards the elaboration of much complex structures such as tunable MEM filters. High quality factors and very low loss up to millimeter-wave frequencies are expected.

\section{REFERENCES}

[1]. Z. Yao and al.: "Micromachined low-loss microwave switches". IEEE Journal of MEMS, vol. 8, $\mathrm{n}^{\circ}$ 2, June 1999, pp. 129-134

[2]. E. Brown : "RF-MEMS switches for reconfigurable integrated circuits". IEEE MTT, vol. 46, $\mathrm{n}^{\circ} 11$, November 1998, pp. 1868-1880

[3]. C. Goldsmith and al.: "Characteristics of micromachined switches at microwave frequencies". IEEE MTT-S Digest, 1996, pp. 1141-1144

[4]. J. Muldavin, G. Rebeiz : "High isolation CPW MEMS shunt switches - Part 1 : Modeling". IEEE MTT, vol. 48, n 6, June 2000, pp. 1045-1052

[5]. K. Grenier, P. Pons, T. Parra, R. Plana, J. Graffeuil : "Silicon microelectromechanical systems for millimeterwave applications". Proc of DTIP of MEMS and MOEMS, Paris, France, May 2000, pp. 354-361

[6]. K. Grenier and al.: "Silicon MEM Technology for Millimeter-Wave Applications". Proc of Microelectronic and MEMS Technologies, Edinburgh, UK, May 2001

[7]. N. Barker, G. Rebeiz : "Distributed MEMS true-time delay phase shifters and wide-band switches". IEEE MTT, vol. 46, n 11, November 1998, pp. 1881-1889 